Ingeniare. Revista chilena de ingeniería, vol. 17 No 2, 2009, pp. 175-181

\title{
SELECCIÓN DETERMINÍSTICA Y CRUCE ANULAR EN ALGORITMOS GENÉTICOS: APLICACIÓN A LA PLANIFICACIÓN DE UNIDADES TÉRMICAS DE GENERACIÓN
}

\section{DETERMINISTIC SELECTION AND ANNULAR CROSSOVER IN GENETIC ALGORITHMS: AN APPLICATION TO THE PLANNING OF THERMAL GENERATING UNITS}

\author{
Boris Pavez-Lazo $^{1} \quad$ Jessica Soto-Cartes $^{1} \quad$ Carlos Urrutia $^{1} \quad$ Millaray Curilem $^{1}$ \\ Recibido 30 de noviembre de 2008, aceptado 4 de junio de 2009 \\ Received: November 30, 2008 Accepted: June 4, 2009
}

\begin{abstract}
RESUMEN
Uno de los problemas fundamentales de los algoritmos genéticos (AG) es la convergencia prematura a un óptimo local, debido principalmente a que los mejores individuos tienden a adueñarse de las características genéticas de la población, disminuyéndose la diversidad. Además, cuando la representación cromosómica es lineal, el cruzamiento es sensible a la codificación, ya que los extremos en este tipo de cromosoma pueden cambiar, con una probabilidad muy baja, sólo si son mutados. En este trabajo se aplica al problema de planificación de unidades térmicas de generación. Un AG que utiliza un operador determinista en la selección de individuos, donde se permite a individuos menos aptos perdurar de una generación a otra y un operador de cruce, llamado Cruce Anular, para generar nuevos individuos. Con ambos mecanismos se permite una mayor diversidad de la población y que el algoritmo sea menos dependiente de la codificación del cromosoma. Estos operadores garantizan la convergencia a una solución óptima, pero no garantizan rapidez de la convergencia. Sin embargo, los resultados obtenidos muestran que estos operadores alcanzan una convergencia más rápida y de mejor calidad que cuando se usan operadores estándares.
\end{abstract}

Palabras clave: Planificación de unidades térmicas, algoritmos genéticos, cruce anular.

\section{ABSTRACT}

One of the fundamental problems of genetic algorithms $(G A)$ is the premature convergence to a local optimum due mainly to the fact that the best individuals tend to take over the genetic characteristics of the population, reducing diversity. Moreover, when the chromosome has a linear representation, the crossover is sensitive to the chromosome codification, as its head and tail change with a very low probability, only if they are affected by mutation. In this work we propose an approach to the problem of thermal generating units scheduling, using a GA which combines a deterministic selection operator with an Annular Crossover operator to generate new individuals. Both mechanisms allow greater diversity of the population and more independence from the codification of the chromosome. These operators guarantee the convergence to an optimal solution, although there is no guarantee of rapid convergence. However, the results show that these operators have a faster convergence and reach a better performance when compared to standard GA operators.

Keywords: Thermal generating units scheduling, genetic algorithms, annular crossover.

\section{INTRODUCCIÓN}

La correcta asignación de unidades generadoras de energía eléctrica tiene un importante rol en la operación económica de cualquier tipo de mercado eléctrico en funcionamiento. Los ahorros económicos junto con el uso eficiente de los recursos energéticos hacen que nuevas propuestas metodológicas sigan investigándose [1,2].

La solución al problema de asignación de unidades ha sido abordada por técnicas de optimización determinísticas, como Relajación Lagrangeana [3-5] la cual resulta muy

\footnotetext{
1 Universidad de La Frontera. Departamento de Ingeniería Eléctrica. Casilla 54-D. Temuco, Chile. E-mail: bpavez@ufro.cl; jessica.soto@gmail.com; curru003@pinhue.ufro.cl; millaray@ufro.cl
} 
eficiente en sistemas de gran tamaño. Por su compatibilidad con este tipo de problemas, técnicas metaheurísticas [6-8] como los Algoritmos Genéticos (AG) han sido propuestas en [9-13]. También, técnicas híbridas [14,16] que toman lo mejor de las técnicas determinísticas y de las técnicas metaheurísticas han sido utilizadas para mejorar la búsqueda de la solución y los tiempos de ejecución.

Los AG se caracterizan por ser una técnica de optimización global que trabaja bien y eficientemente con funciones objetivo complejas. Sin embargo, una de las desventajas de los AG es la convergencia prematura, porque cuando la selección de individuos se basa en la aptitud, la información genética de los mejores individuos tiende a dominar las características genéticas de la población. Otra desventaja está dada por la representación cromosómica en forma de cadena lo que origina que durante el cruce los extremos del cromosoma tiendan a mantener inalterada su información genética solo siendo posible lograr una modificación mediante el operador de mutación.

Para evitar estas desventajas se propone el uso de un AG propuesto en [17]. Este AG está basado en una selección determinista en la cual todos los individuos de la población son seleccionados como padres en razón de una estrategia establecida, y un operador de cruce anular el cual permite generar cambios de información en los extremos del cromosoma.

Mediante el AG propuesto, el problema de asignación de unidades es resuelto para un periodo de planificación de 24 horas de un sistema de 38 unidades y de la misma forma que en [13] una reserva de potencia del $11 \%$ es considerada. Este sistema de prueba representa el conjunto de unidades térmicas del sistema de potencia de Taiwan.

\section{PLANIFICACIÓN DE UNIDADES TÉRMICAS}

La función objetivo a minimizar y las restricciones asociadas con el problema de planificación de unidades térmicas son las siguientes:

\section{Función Objetivo}

El modelo matemático usado como función objetivo de la planificación de unidades térmicas es:

$$
F=\sum_{h=1}^{H} \sum_{n=1}^{N}\left\{F C_{n}^{h}+S U_{n}^{h}+S D_{n}\right\}
$$

Donde $H$ representa el número de intervalos que dividen el periodo de estudio y $N$ es el número de unidades térmicas del sistema.

$F$ representa el total de los costos de producción para el periodo de planificación en estudio. Esta función de costos incluye costos de combustible, costos de parada y costos de partida de las unidades térmicas.

La función de costos de combustible de la unidad $n$ en la hora $h$ normalmente se representa por una ecuación cuadrática como:

$$
F C_{n}^{h}=a_{n} P_{n}^{2}+b_{n} P_{n}+c_{n} \quad h \in[1, H]
$$

Los costos de partida son dependientes de la cantidad de horas que la unidad ha estado apagada $\left(\right.$ TOff $\left._{n}\right)$, y se pueden representar como:

$$
S U_{n}^{h} \begin{cases}H S_{n} & \text { si } \text { TOff }_{n} \leq \text { TCold }_{n} \\ C S_{n} & \text { otro caso }\end{cases}
$$

Donde $H S_{n}$ es el costo de partida en caliente, y $C S_{n}$ es el costo de partida en frío, TCold $_{n}$ es el número de horas que la caldera de la unidad $n$ tarda en enfriarse y se calcula como:

$$
T_{\text {Cold }, n}=T d n_{n}+\mathrm{CSH}_{n}
$$

Donde, $T d n_{n}$ es el tiempo mínimo de apagado de la unidad $n$ y $\mathrm{CSH}_{n}$ son las horas que la unidad toma al partir desde frío.

Los costos de parada generalmente son considerados como un valor constante. El sistema de prueba utilizado en este artículo tiene costos de parada igual a cero en todas las unidades.

\section{Restricciones del sistema}

La potencia suministrada por todas las unidades asignadas debe ser igual a la demanda en la hora $h$, es decir:

$$
\sum_{n=1}^{N} P_{n}=D^{h}, \quad h \in[1, H]
$$

Además, la suma de las potencias máximas generadas por las unidades asignadas debe ser mayor o igual a la 
demanda más el nivel de reserva en potencia requeridas para la hora $h$.

$$
\sum_{n=1}^{N} \operatorname{PMax}_{n} \geq D^{h}+R^{h}, \quad h \in[1, H]
$$

Por otro lado, para considerar las condiciones de mínima demanda es necesario que la potencia mínima generada por las unidades asignadas sea menor o igual a la demanda en la hora $h$. Esta restricción se formula como:

$$
\sum_{n=1}^{N} \operatorname{PMin}_{n} \geq D^{h}, \quad h \in[1, H]
$$

Además de las restricciones impuestas por el sistema, se deben considerar las restricciones técnicas que imponen las unidades de tipo térmicas.

Una de estas restricciones es que el total de horas que la unidad ha estado funcionando $\left(\mathrm{TO}_{n}\right)$ o ha estado fuera de servicio $\left(\operatorname{TOff}_{n}\right)$ debe ser superior o igual al mínimo tiempo de encendido $\left(\operatorname{Tup}_{n}\right)$ o al mínimo tiempo de apagado $\left(T d n_{n}\right)$, respectivamente. Este tipo de restricciones se representan como:

$$
\begin{aligned}
& \operatorname{TOn}_{n} \geq \operatorname{Tup}_{n} \quad, n \in N \\
& \operatorname{TOff}_{n} \geq \operatorname{Tdn}_{n} \quad, n \in N
\end{aligned}
$$

Cuando una unidad es asignada, la potencia capaz de generar debe estar entre un valor mínimo y un valor máximo de potencia, es decir:

$$
\operatorname{PMin}_{n} \leq P_{n} \leq \operatorname{PMax}_{n}, \quad n \in N
$$

Finalmente, en la búsqueda de una solución para el horizonte de tiempo en estudio se deben considerar los estados iniciales de las unidades. Es decir, se deben tomar en cuenta para el período inicial de la planificación las horas que las unidades han estado encendidas y las horas que las unidades han estado apagadas.

\section{ALGORITMO GENÉTICO PROPUESTO}

\section{Generalidades}

Los algoritmos genéticos son técnicas de búsqueda robustas inspiradas en la genética y en los procesos de selección natural que se manifiestan entre individuos que compiten en un mismo ambiente. Los individuos mejor adaptados al ambiente tienden a transmitir su información genética a las futuras generaciones y así perpetuar sus habilidades.

Una de las ventajas que brindan los algoritmos genéticos en problemas de optimización es que no necesitan mayor información que la proporcionada por la función objetivo del problema. Con esta técnica se aísla el proceso de búsqueda de soluciones de las características de la función objetivo y de las restricciones asociadas.

El algoritmo comienza por la creación de un conjunto de estructuras codificadas llamadas cromosomas (soluciones) que componen la población inicial. El criterio que valora la calidad de cada cromosoma es el entregado por el Fitness que corresponde a la evaluación de cada individuo mediante la función objetivo. Conocida la calidad de los individuos de la población estos son sometidos a un proceso de selección donde los individuos mejor evaluados tienen mayores probabilidades de ser elegidos como padres para el intercambio de información genética llamado cruce (proceso análogo a la reproducción biológica). Luego un porcentaje de los hijos (individuos generados en el cruce) son sometidos al proceso de mutación donde en forma aleatoria se genera un cambio en el cromosoma. Este proceso de mutación proporciona mayor diversidad en los individuos de la población.

Completados los procesos de cruce y mutación se genera la nueva población que reemplaza a la población original. Esto debe ser repetido hasta cumplir con algún criterio de convergencia definido para el problema. Cada uno de estos ciclos es conocido como Generación.

\section{Selección Determinista}

Tradicionalmente, la selección está basada en la aptitud de los individuos, es decir, los individuos más aptos tienen mayor probabilidad de ser escogidos para la reproducción. En esta propuesta se usa una selección determinista llamada Vasconcelos [17] la cual impone una estrategia en la cual los individuos más aptos se cruzan con los individuos menos aptos de la población.

De esta forma, para una población de $K$ individuos ordenados de acuerdo a su aptitud en orden descendente como se representa en la figura 1, los pares de padres que pasarán al operador de cruce serán $(1, K),(2, K$-1) hasta el último par formado por $(K / 2, K / 2+1)$. 


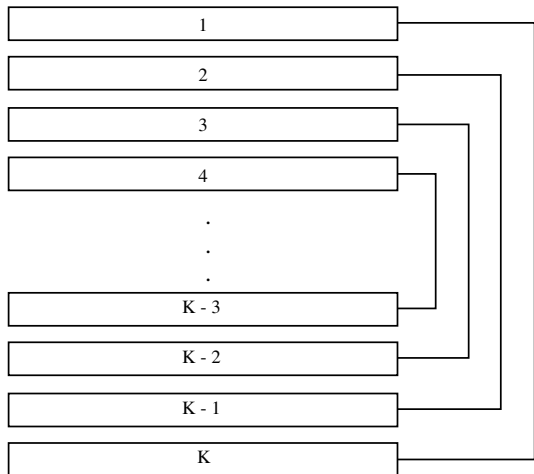

Figura 1. Selección determinista.

\section{Cruce Anular}

Una vez seleccionados dos individuos cuyo cromosoma es tradicionalmente representado mediante una cadena como en la figura 2.a, estos son sometidos al proceso de cruce donde se genera un intercambio de información. Normalmente este proceso se efectúa mediante la división de los cromosomas en un punto aleatoriamente establecido. Luego, se intercambian los segmentos finales de cada cromosoma generándose así dos nuevos descendientes.

\section{\begin{tabular}{|l|l|l|l|l|l|l|}
\hline A & B & C & D & E & F & G \\
\hline
\end{tabular}}

a)

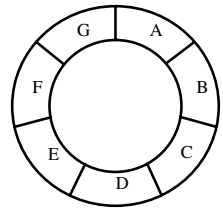

b)
Figura 2. Representación cromosómica.

a) cadena b) anillo.

A diferencia de lo anterior, este trabajo considera que el cromosoma es un anillo tal como se observa en la figura 2.b. De esta forma, para el cruce anular se define un número $C_{l}$ que indica el lugar o punto de cruce. Este número se encuentra en el rango [1, $L-1]$, donde $L$ es la longitud del cromosoma. Además, es necesario definir un número que establezca la longitud del semianillo $C_{s}$ que se intercambia durante el cruzamiento. Por semianillo nos referimos al sector del anillo cuyo largo se encuentra en el rango $[1, L / 2]$.

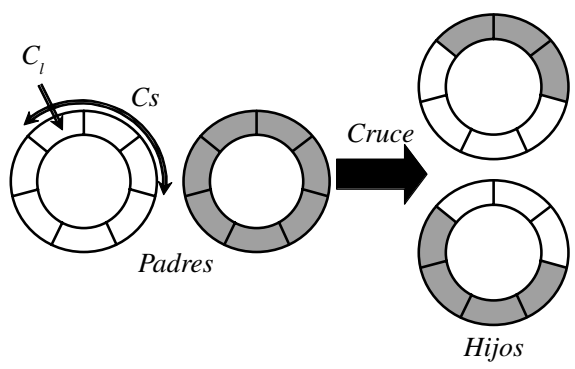

Figura 3. Representación del Cruce Anular.

\section{APLICACIÓN Y RESULTADOS}

\section{Representación de la solución}

La solución al problema de asignación de unidades se puede representar como una matriz de orden $\mathrm{NxH}$ como se muestra en la figura 4 , donde $N$ es el número de unidades generadoras y $H$ el número de horas del periodo en estudio. Se utiliza una codificación binaria donde el 1 representa que la unidad se encuentra encendida $(\mathrm{ON})$ y el 0 representa que la unidad está apagada (OFF).

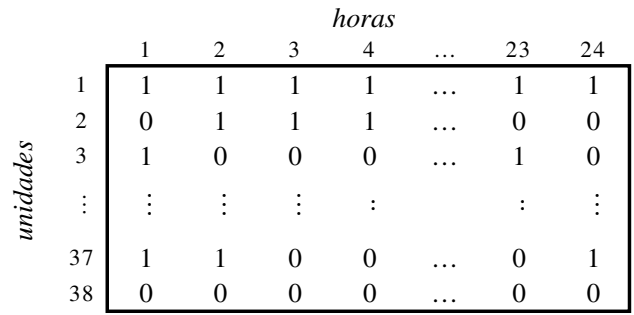

Figura 4. Representación de la solución.

\section{Selección}

Para una población de 50 individuos ordenados de acuerdo a su aptitud en orden descendente. Los pares de padres que son sometidos al proceso de cruce, son elegidos mediante la selección determinista descrita en sección 3.2. Usando la representación matricial este proceso de selección puede ser representado como lo muestra la figura 5.

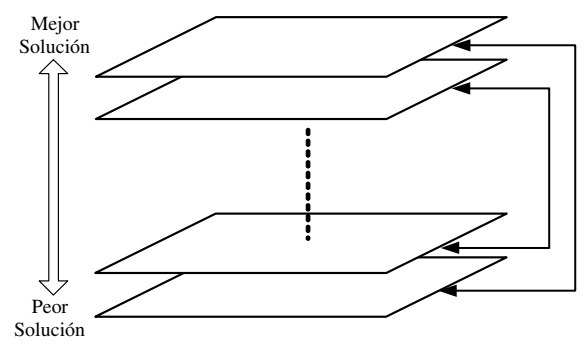

Figura 5. Representación del proceso de selección.

\section{Mecanismo de Cruce Anular}

Para el operador de cruce anular propuesto es necesario realizar los siguientes pasos.

Paso 1. De cada padre seleccionado, elegir de forma aleatoria y uniformemente distribuida sobre $[1, N]$, una unidad $n$ y una unidad $m$.

Paso 2. Definir las planificaciones de las unidades elegidas mediante una representación cromosómica anular como figura 6. 

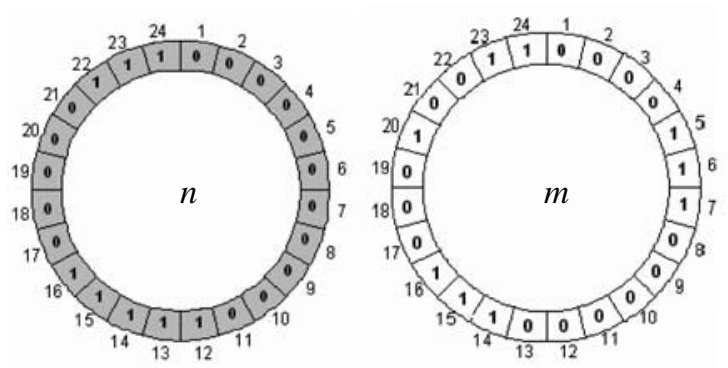

Figura 6. Representación en anillo de la planificación de las unidades $n$ y $m$.

Paso 3. Generar el punto de cruce $C_{l}$ de forma aleatoria

Paso 4. Seleccionar de forma aleatoria la longitud del semianillo $C_{s}$. En este caso $H$ es el largo del período de planificación, es decir 24 hrs. Si $C_{l}=22$ y $C_{s}=9$, Fig. 7 muestra los semianillos resultantes.
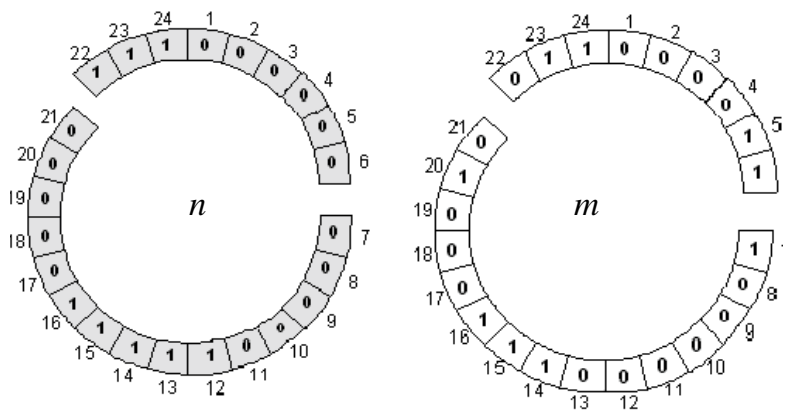

Figura 7. Semianillos para el cruce anular.

Paso 5. Generar los nuevos descendientes intercambiando la información genética de los semianillos de los individuos padres. La figura 8 muestra la información genética de los nuevos descendientes para las unidades $n$ y $m$.
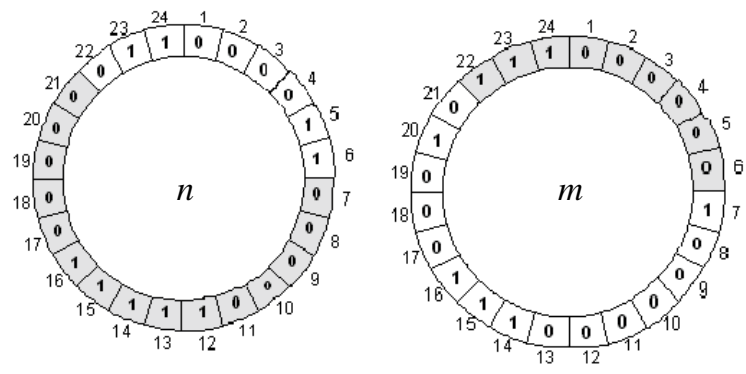

Figura 8. Planificaciones de las unidades $n$ y $m$ después del cruce.

Paso 6. Incorporar los nuevos descendientes a la población de hijos.
Paso 7. Fin del cruce. Si se completa el número de pares de individuos de la población, el operador de cruce anular termina.

\section{Mutación}

En esta propuesta, se define una probabilidad de mutación $(P m)$, que se refiere a la probabilidad de modificar la información genética del cromosoma sometido al operador mutación. Esta modificación en la información genética se realiza de acuerdo a los siguientes pasos:

Paso 1. Elegir una posición o locus de la matriz.

\begin{tabular}{|c|c|c|c|c|c|c|c|}
\hline & & & & ora & & & \\
\hline & 1 & 2 & 3 & 4 & & 23 & 24 \\
\hline 1 & 1 & 1 & 1 & 1 & $\ldots$ & 1 & 1 \\
\hline 2 & 0 & 1 & 1 & 1 & $\ldots$ & 0 & 0 \\
\hline 3 & 1 & 0 & 0 & 0 & $\ldots$ & 1 & 0 \\
\hline$\vdots$ & $\vdots$ & $\vdots$ & $\vdots$ & : & & : & $\vdots$ \\
\hline 37 & 1 & 1 & 0 & 0 & $\ldots$ & 0 & 1 \\
\hline 38 & 0 & 0 & 0 & 0 & $\ldots$ & 0 & 0 \\
\hline
\end{tabular}

Figura 9. Individuo a mutar. Elemento destacado corresponde a la posición a modificar.

Paso 2. Si el bit corresponde a un 1, se cambia por un 0 , y en caso contrario, si el bit corresponde a un 0 se cambia por un 1 .

\begin{tabular}{|c|c|c|c|c|c|c|c|}
\hline & & & & ora & & & \\
\hline & 1 & 2 & 3 & 4 & $\ldots$ & 23 & 24 \\
\hline 1 & 1 & 1 & 1 & 1 & $\ldots$ & 1 & 1 \\
\hline 2 & 0 & 1 & 1 & 1 & $\ldots$ & 0 & 0 \\
\hline 3 & 1 & 0 & 1 & 0 & $\ldots$ & 1 & 0 \\
\hline$\vdots$ & $\vdots$ & $\vdots$ & $\vdots$ & : & & : & $\vdots$ \\
\hline 37 & 1 & 1 & 0 & 0 & $\ldots$ & 0 & 1 \\
\hline 38 & 0 & 0 & 0 & 0 & $\ldots$ & 0 & 0 \\
\hline
\end{tabular}

Figura 10. Individuo mutado.

Paso 3. El individuo mutado se reincorpora a la población de descendientes.

Paso 4. Fin de la Mutación. Si se han mutado los individuos el operador mutación termina.

\section{Nueva Población}

Para crear la nueva población se juntan la población de padres y la población de hijos, se evalúan todos los individuos y se eligen los $K$ mejores, los cuales formarán una nueva población para la siguiente generación. Este tipo de estrategia es conocida como Elitismo Máximo. 


\section{Resultados}

Para tener una muestra confiable se ejecuta 20 veces el algoritmo propuesto. Se usa un sistema de prueba de 38 unidades para un periodo de estudio de 24 horas y de la misma forma que en [13] una reserva de potencia del $11 \%$ es considerada.

De los resultados encontrados tras las 20 ejecuciones, la mejor solución es M\$197.2 y la peor fue de M\$200.8. La solución promedio del total de ejecuciones fue M\$ 198.3. En la figura 11 se muestra la forma en la cual converge a la mejor solución el algoritmo propuesto. Se puede observar que alrededor de las 3.000 generaciones una solución óptima es encontrada y en las generaciones siguientes pequeños cambios respecto a esta solución son obtenidas.

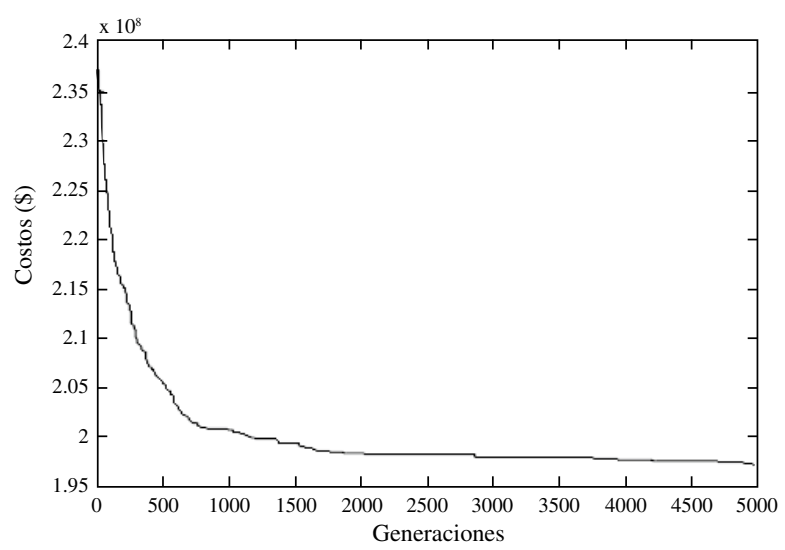

Figura 11. Convergencia a la mejor solución encontrada.

En tabla 1 se muestran las convergencias promedios obtenidas con el AG propuesto y comparados con los obtenidos en [13] donde el AG utiliza una selección Ruleta y un operador de cruce de tipo lineal.

Tabla 1. Comparación de costos en relación al número de generaciones.

\begin{tabular}{|l|c|c|c|c|c|c|c|}
\cline { 2 - 8 } \multicolumn{1}{c|}{} & \multicolumn{7}{c|}{ Generaciones } \\
\cline { 2 - 8 } \multicolumn{1}{c|}{} & 200 & 400 & 800 & 1.200 & 1.600 & 1.900 & 3.000 \\
\hline Sun [13] & 211,1 & 209,7 & 208,2 & 207,0 & 206,5 & 206,3 & - \\
\hline Propuesta & 221,7 & 207,3 & 200,9 & 199,9 & 198,8 & 198,3 & 197,9 \\
\hline
\end{tabular}

Se puede observar que a partir de las 400 generaciones el algoritmo propuesto empieza a entregar mejores soluciones.

\section{CONCLUSIONES}

El AG propuesto permite gracias al operador de selección determinista una mayor diversidad entre los individuos ya que de igual manera ocupa la información genética de individuos menos aptos complementando las características de los individuos más aptos. Además, el operador de cruza anular permite una mayor probabilidad de intercambio de la información genética en los periodos iniciales y finales de la planificación de 24 hrs.

Los resultados encontrados y comparados con los obtenidos por otro AG permiten validar la aplicación del AG propuesto al problema de planificación de unidades térmicas de generación, mejora la convergencia y aumenta la calidad de la mejor solución encontrada, reflejada en una disminución del costo de producción.

\section{AGRADECIMIENTOS}

Boris J. Pavez-Lazo, agradece el apoyo financiero dado por la Universidad de La Frontera a través del proyecto DIUFRO 07-0049.

Jessica Soto-Cartes agradece el apoyo financiero de la Universidad de La Frontera a través del proyecto DIUFRO 07-0049 y al programa de Magíster en Ciencias de la Ingeniería mención Ingeniería Eléctrica de la Universidad de La Frontera.

\section{REFERENCIAS}

[1] N.P. Padhy. "Unit Commitment - A Bibliographical Survey". IEEE Transactions on Power Systems. Vol. $19 \mathrm{~N}^{\circ}$ 2, pp. 1196-1205. May 2004.

[2] H.Y. Yamin. "Review on methods of generation scheduling in electric power systems". Electric Power Systems Research. Vol. 69, pp. 227-248. 2004.

[3] S. Vermin, K. Imhof and S. Mukherjee. "Implementation of Lagrangean Relaxation Based Unit Commitment Problem". IEEE Transaction on Power Systems. Vol. 4, pp. 1373-1379. October 1989.

[4] Q. Zhai, X. Guan and J. Cui. "Unit commitment with identical units: successive subproblem solving method based on lagrangian relaxation". IEEE Transactions on Power Systems. Vol. 17, pp. 12501257. November 2002. 
[5] W. Ongsakul and N. Pertcharaks. "Unit commitment by enhanced adaptive lagrangian relaxation”. IEEE Transactions on Power Systems. Vol. 19, pp. 620628. February 2004.

[6] H. Mantawy, Y.L. Abdel-Magid and S.Z. Selim. "A Simulated Annealing Algorithm for Unit Commitment". IEEE Transactions on Power Systems. Vol. 13, pp. 197-204. February 1998.

[7] D.N. Simopoulus, S.D. Kavatza and C.D. Vournas. "Unit Commitment by Enhanced Simulating Annealing Algorithm". IEEE Transaction on Power Systems. Vol. 21, pp. 68-76. February 2006.

[8] I.G. Damousis, A.G. Bakirtzis and P.S. Dokopolous. "A solution to the unit commitment using integercoded genetic algorithm". IEEE Transactions on Power Systems. Vol. 19, pp. 1165-1172. May 2004.

[9] C. Dang and M. Li. "A floating-point genetic algorithm for solving the unit commitment problem". European Journal of Operational Research. Vol. 181, pp. 1370-1395. 2007.

[10] G. Dudek. "Unit commitment by genetic algorithm with specialized search operators". Electric Power Systems Research. Vol. 72, pp. 299-308. 2004.
[11] K.S. Swarup and S. Yamashiro. "Unit commitment solution methodology using genetic algorithm". IEEE Transactions on Power Systems. Vol. 17, pp.87-91. February 2002.

[12] L. Sun, Y. Zhang and C. Jiang. "A matrix realcoded genetic algorithm to the unit commitment problem”. Electric Power Systems Research. Vol. 76, pp. 716-728. 2006.

[13] A.V.T. Aruldoss, J.A. Ebenezer. "A tabu search based hybrid optimization approach for a fuzzy modelled unit commitment problem". Electric Power System Research. Vol. 76, pp. 413-425. 2006.

[14] N.P. Padhy. "Unit commitment using hybrid models: a comparative study for dynamic programming, expert system, fuzzy system and genetic algorithms". International Journal of Electrical Power \& Energy Systems. Vol. 23, pp. 827-836. 2001.

[15] S.W. Yin-Wa. "Hybrid simulated annealing/ genetic algorithm approach to short-term hydrothermal scheduling with multiple thermal plants". International Journal of Electrical Power \& Energy Systems. Vol. 23 No 7, pp. 565-575. 2001.

[16] A. Kuri. "Pattern Recognition via Vasconcelos' Genetic Algorithm". Lecture Notes in Computer Science. Vol. 3287, pp. 328-335. 2004. 